\title{
Candidate stress biomarkers for queen failure diagnostics
}

\author{
Alison McAfee ${ }^{1 *}$ D, Joseph Milone ${ }^{1}$, Abigail Chapman², Leonard J. Foster ${ }^{2}$, Jeffery S. Pettis ${ }^{3}$ and David R. Tarpy ${ }^{1}$
}

\begin{abstract}
Background: Queen failure is a persistent problem in beekeeping operations, but in the absence of overt symptoms it is often difficult, if not impossible, to ascertain the root cause. Stressors like heat-shock, cold-shock, and sublethal pesticide exposure can reduce stored sperm viability and lead to cryptic queen failure. Previously, we suggested candidate protein markers indicating heat-shock in queens. Here, we further investigate these heat-shock markers and test new stressors to identify additional candidate protein markers.

Results: We found that heat-shocking queens for upwards of $1 \mathrm{~h}$ at $40^{\circ} \mathrm{C}$ was necessary to induce significant changes in the two strongest candidate heat-shock markers, and that relative humidity significantly influenced the degree of activation. In blind heat-shock experiments, we tested the efficiency of these markers at assigning queens to their respective treatment groups and found that one marker was sufficient to correctly assign queens $75 \%$ of the time. Finally, we compared cold-shocked queens at $4{ }^{\circ} \mathrm{C}$ and pesticide-exposed queens to controls to identify candidate markers for these additional stressors, and compared relative abundances of all markers to queens designated as 'healthy' and 'failing' by beekeepers. Queens that failed in the field had higher expression of both heat-shock and pesticide protein markers, but not cold-shock markers.

Conclusions: This work offers some of the first steps towards developing molecular diagnostic tools to aid in determining cryptic causes of queen failure. Further work will be necessary to determine how long after the stress event a marker's expression remains elevated, and how accurate these markers will be for field diagnoses.
\end{abstract}

Keywords: Honey bees, Queens, Sperm viability, Biomarkers, Proteomics, Stressors, Spermatheca

\section{Background}

According to surveys in Canada [1] and the US [2, 3], one of the most frequently reported causes of honey bee (Apis mellifera) colony failure is 'poor queens.' Unfortunately, the underlying factors leading to queen failure are usually difficult, if not impossible, to determine. For example, previous research has found that heat-shock, cold-shock, and pesticide exposure all decrease the viability of sperm stored in queen spermathecae $[4,5]$, which would theoretically lead to the same colony-level symptoms: inconsistent brood patterns, poor population

\footnotetext{
* Correspondence: alison.n.mcafee@gmail.com

'Department of Entomology \& Plant Pathology, North Carolina State University, Raleigh, North Carolina, USA

Full list of author information is available at the end of the article
}

build-up, and sometimes atypical drone-laying. If beekeepers cannot diagnose the root cause of queen failure, they are less equipped to identify the source of stress and are unable to make evidence-based management decisions to mitigate or eliminate the stress in the future.

In colonies, honey bees are normally adept at thermoregulating [6], but queens are vulnerable to heat-shock and cold-shock events during shipping $[4,7]$, where they are regularly transported long distances via ground or air cargo in poorly thermoregulated environments [8]. In a colony, worker bees can try to lessen the effects of overheating by collecting water and fanning to achieve evaporative cooling, or by heat-shielding, using their own bodies as heat-blocking insulation [6,9]. Likewise, they can mitigate chilling by vibrating their wing

(c) The Author(s). 2020 Open Access This article is licensed under a Creative Commons Attribution 4.0 International License, which permits use, sharing, adaptation, distribution and reproduction in any medium or format, as long as you give appropriate credit to the original author(s) and the source, provide a link to the Creative Commons licence, and indicate if changes were made. The images or other third party material in this article are included in the article's Creative Commons licence, unless indicated otherwise in a credit line to the material. If material is not included in the article's Creative Commons licence and your intended use is not permitted by statutory regulation or exceeds the permitted use, you will need to obtain permission directly from the copyright holder. To view a copy of this licence, visit http://creativecommons.org/licenses/by/4.0/. The Creative Commons Public Domain Dedication waiver (http://creativecommons.org/publicdomain/zero/1.0/) applies to the data made available in this article, unless otherwise stated in a credit line to the data. 
muscles to generate heat [6]. However, in small queen cages used for shipping, there is often limited (if any) water, poor ventilation, and too few workers to effectively cool or heat the queen. We have previously included temperature loggers in long distance queen shipments and found that both hot and cold temperature spikes regularly occur $[4,7]$. Some commercial queen suppliers include temperature loggers in their shipments as a quality control measure, but these are typically only large-order, international deliveries, and it is up to the discretion of the supplier. Worryingly, some evidence suggests that temperature spikes can even occur inside colonies during extreme heat-waves $[7,10]$ - conditions that are projected to increase in frequency, severity, and duration with climate change [11, 12].

Pesticides from multiple sources (mainly beekeeperapplied miticides and agrochemicals) can accumulate in hive products, creating an additional risk to honey bee health. Since queens are fed a strict diet of worker glandular secretions (i.e, they do not consume potentially contaminated flower products) [13-15], they are normally buffered from direct oral xenobiotic exposure $[16,17]$. However, pesticides (including miticides, fungicides, herbicides, and insecticides) accumulate in wax $[18,19]$ and thus can pose a contact exposure hazard to queens.

A cumulative hazard quotient (HQ) is one way to interpret risk during co-exposures to multiple pesticides simultaneously and employs a solely additive approach to incorporate the dose and respective toxicity of all pesticides in a mixture to estimate hazard [20]. Here, hazard quotients represent the ratio of the expected toxin exposure divided by the $\mathrm{LD}_{50}$ based on adult worker oral exposure (the same approach as reported in Traynor et al. [18]). This method is imperfect, since queen topical exposure will have different toxicity than adult worker oral exposure, but this approach does allow for standardization of mixture doses. Although the biological effects of xenobiotics are not always additive, cumulative hazard quotients provide a framework to assess cocktail risks when interactions between their components are unknown.

In a survey of commercial colonies engaged in agricultural pollination, Traynor et al. found that the mean hazard quotient of the cumulative pesticide 'exposome' in wax was 2155 [18]. Those colonies experiencing 'queen events' (i.e., the colony was queenless, had queen cells, or a virgin queen) had an average wax $\mathrm{HQ}$ of $\sim 3500$, while queenright colonies had average wax HQ of 1700. While this is only a correlational observation, other researchers have found increased frequencies of queen events when colonies were given pollen patties with clothianidin [21] or a mix of clothianidin and thiamethoxam [22], both of which are neonicotinoid pesticides. Neonicotinoids can also reduce stored sperm viability [5], which is a possible mechanism leading to queen failure events.

Our ultimate goal is to identify queen stress biomarkers that can help distinguish different causes of queen failure in a single diagnostic test (Fig. 1). As a first step towards that goal, here we heat-shocked, coldshocked, and pesticide-stressed queens experimentally and compared protein expression profiles of their spermathecae to controls. The spermathecae were chosen because if a queen is sent for laboratory analysis, the spermathecae will likely be dissected to determine sperm viability and sperm count metrics, and we have previously shown that the remaining tissue sample is amenable to proteomics by mass spectrometry. Therefore, it is an economical and biologically relevant choice. We also surveyed protein expression profiles in 125 'imported,' 'healthy,' and 'failed' queens to look for overlap between their expression patterns and the candidate biomarkers. Finally, we conducted a blind heat-shock trial to test the efficacy of using the top two candidate heat-shock biomarkers to assign queens to their relevant treatment groups. This work forms the foundation on which to further develop and refine these candidate queen stress biomarkers for eventual diagnostic use.

\section{Results}

\section{Temperature stress}

Previously, we heat-shocked queens and used comparative proteomics to suggest candidate spermathecal protein markers [7]. To expand our stressor scope, here we also compared cold-shocked queens to controls and investigated the proposed heat-shock markers in more detail through an exposure time-course. Of the 2094 quantified proteins, 21 were differentially expressed between cold-shocked $\left(2 \mathrm{~h}\right.$ at $\left.4{ }^{\circ} \mathrm{C}\right)$ and control queens ( $n=5$ and 7, respectively), and only three of those were upregulated in the cold-shock group (Fig. 2a; Student's $t$ test, $5 \%$ false discovery rate (FDR), Benjamini-Hochberg correction). GO term enrichment by the gene score resampling approach, which does not depend on $p$ value thresholds [23], shows that acyl transferase activity was significantly upregulated with cold-shock, whereas oxidoreductase activity was significantly downregulated, among others (Fig. 2b; 5\% FDR, Benjamini-Hochberg correction). We anticipate that upregulated, rather than downregulated, proteins will generally be the most practical biomarkers because they are less likely to be affected by instrumental limits of detection and should not be as sensitive to false positives (e.g., from sample degradation). Therefore, we selected the top two most significantly upregulated proteins, XP_026296654.1 (leucine-rich repeat transmembrane neuronal protein 4-like) and XP_395122.1 (probable tRNA N6-adenosine 


\section{The Problem \\ Queens can become temperature-stressed during routine shipment and pesticide-stressed from contaminated wax}

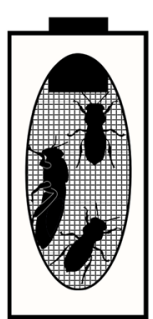

Producer

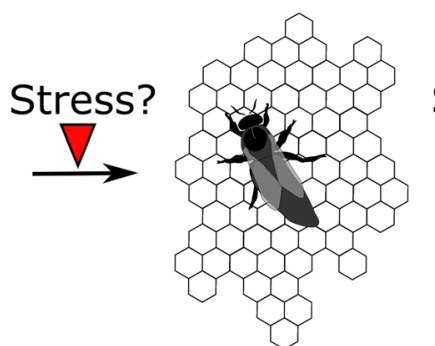

Buyer

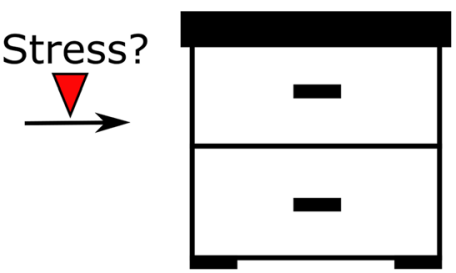

Failed Colony

\section{Develop Reference Stress Signatures}

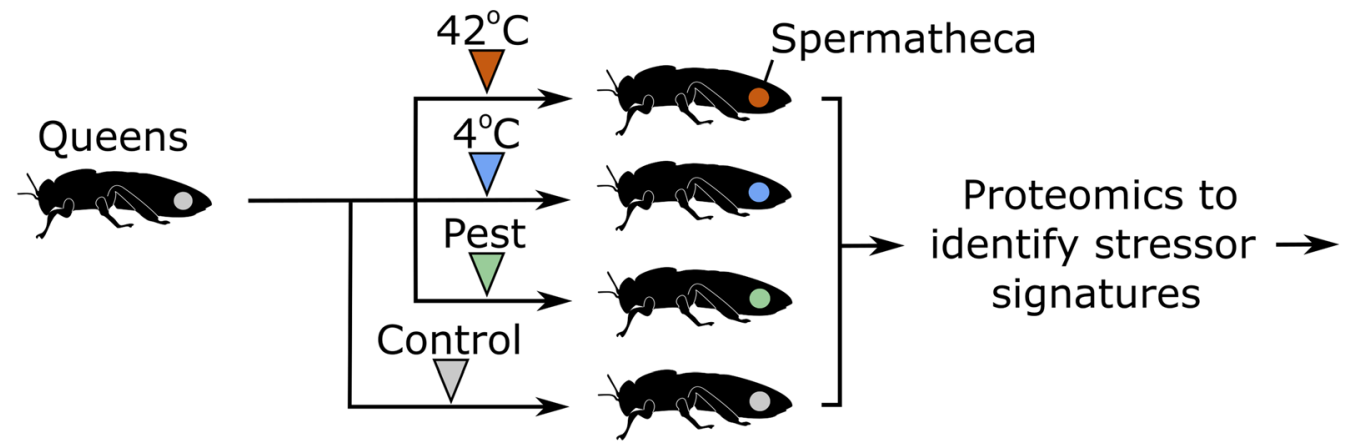

\section{Match Signatures in Failed Queens}
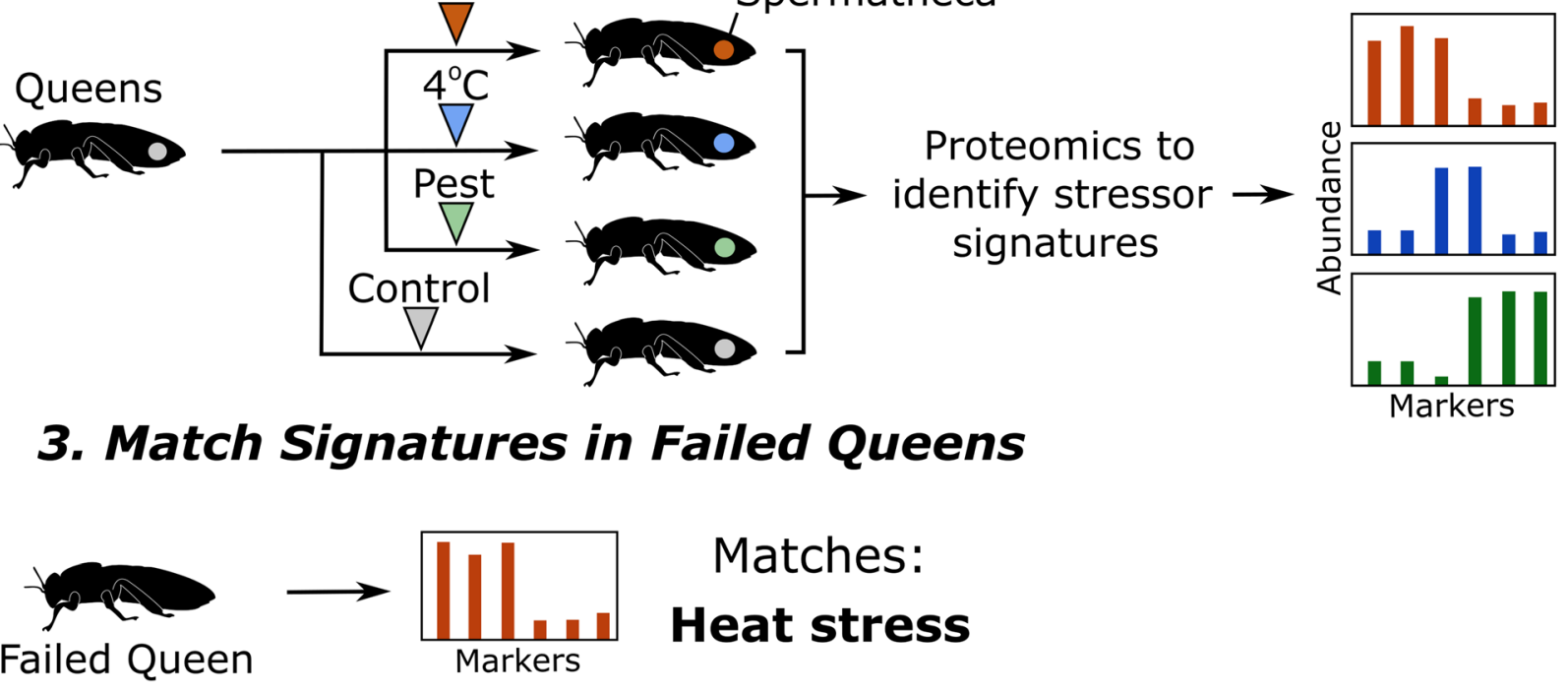

Fig. 1 Schematic of experimental design for identifying queen stress biomarkers. Queen silhouettes adapted from McAfee et al. (2019), reuse permitted under CC BY 4.0

threonylcarbamoyltransferase) as candidate cold-shock biomarkers.

The two most significant candidate heat-shock biomarkers we identified in our previous experiment [7] were the small heat-shock proteins XP_395659.1 and XP_001120006.2, and here we confirm that these two proteins are consistently upregulated across queens from independent origins. Both proteins were again globally differentially expressed after a $2 \mathrm{~h}, 40{ }^{\circ} \mathrm{C}$ exposure relative to negative controls (5\% FDR, Benjamini-Hochberg, $n=7$ each), and this response was significantly higher at $80 \%$ relative humidity ( $\mathrm{RH} ; n=7)$ compared to $40 \% \mathrm{RH}$ (Fig. 2e; $n=6$; XP_001120006.2: $\mathrm{df}=11, p=0.0055$, $\mathrm{T}_{\text {stat }}=3.44 ;$ XP_395659.1: $\mathrm{df}=11, p=0.0075, \mathrm{~T}_{\text {stat }}=$ $3.26)$. Holding the $\mathrm{RH}$ at a constant $40 \%$, expression of $\mathrm{XP} \_001120006.2$ (linear regression, $\mathrm{df}=34, p=7.9 \times$ $\left.10^{-8}, \mathrm{~F}=47.0\right)$ and XP_395659.1 $\left(\mathrm{df}=33, p=5.1 \times 10^{-6}\right.$, $\mathrm{F}=29.9$ ) significantly depended on exposure duration, with clear differences not emerging until the $1 \mathrm{~h}$ time point.

\section{Pesticide stress}

Temperature is one of many stress factors that can influence queen quality, as pesticide exposure can also reduce sperm viability and lead to queen failure [5, 21, 22]. To evaluate effects of pesticide exposure on spermathecal protein expression, we applied (to the thorax) $2 \mu \mathrm{l}$ of either acetone alone, acetone with $20 \mathrm{ppb}$ imidacloprid (a neonicotinoid insecticide), a pesticide "cocktail" (which does not contain any neonicotinoids) diluted in acetone to achieve a hazard quotient of $\sim 511$ (Table 1 ). This HQ is considerably lower than the mean wax HQ in commercial colonies (2155), which was intentional because we expect the transfer of toxins from wax to the queen to be inefficient and we also sought to examine the queen biomarkers resulting from sublethal pesticide exposure. The imidacloprid dose is also an equivalent amount to the lowest dose Chaimanee et al. [5] tested on queens ( $2 \mathrm{ul}$ of $20 \mathrm{ppb}$ imidacloprid applied to the thorax), which they found caused a significant drop in 


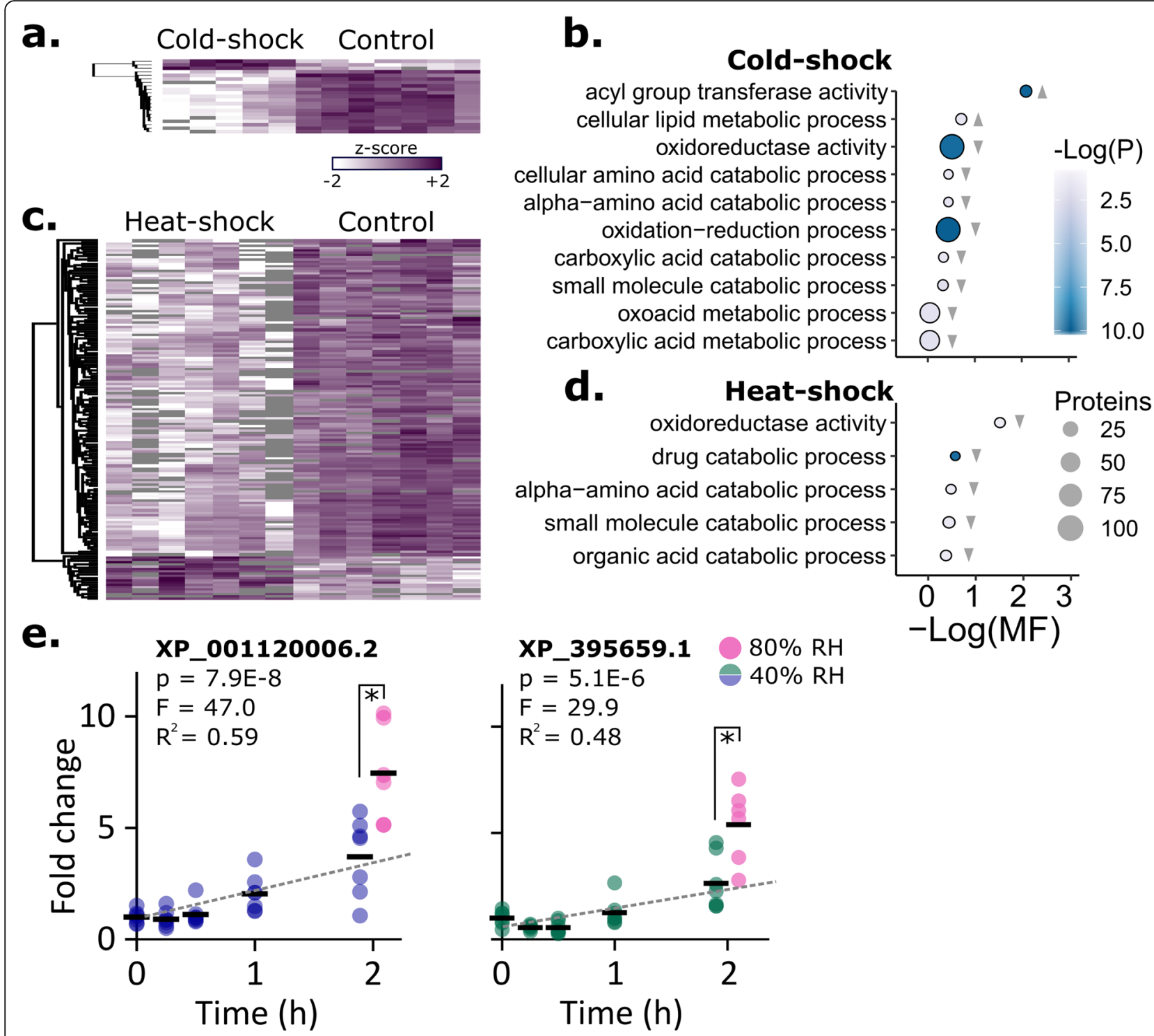

Fig. 2 Effects of heat-shock and cold-shock on spermathecal protein abundance. a Protein expression changes induced by cold-shock at $4{ }^{\circ} \mathrm{C}$ for $2 \mathrm{~h}$ (only significant proteins surviving 5\% FDR threshold by the Benjamini-Hochberg method are shown). The two most significantly upregulated proteins are XP_026296654.1 (leucine-rich repeat transmembrane neuronal protein) and XP_395122.1 (probable tRNA N6-adenosine

threonylcarbamoyltransferase). Each column represents the spermatheca from one queen. b GO term enrichment analysis. Grey triangles indicate the direction of expression driving the enrichment (relative to control). Only GO terms passing a 5\% false discovery cut-off are shown (BenjaminiHochberg method). MF stands for multifunctionality score (higher values are less multifunctional). Circle size is proportional to the number of proteins belonging to the $\mathrm{GO}$ term. c Protein expression changes induced by heat-shock at $40^{\circ} \mathrm{C}$ for $2 \mathrm{~h}$. $\mathbf{d}$ Enrichment analysis. e Temporal changes in expression of two previously identified candidate heat-shock biomarkers. Expression was significantly influenced by time and relative humidity $(\mathrm{RH})$. Black bars indicate the mean

sperm viability. No queens died during these experiments.

We found stark differences between cocktail-treated and untreated queens, with intermediate effects of acetone alone (Fig. 3a; $n=7,10$, and 8 , respectively). The effect of acetone in our solvent control group was significant (653 proteins were differentially expressed between acetone-treated and untreated queens at 5\% FDR, Benjamini-Hochberg correction), indicating that, although commonly used, this organic carrier is far from benign. However, our interest is in proteins specifically altered by the pesticide treatments.

Of 2408 quantified proteins, 61 were differentially expressed between the pesticide cocktail-treated and acetone-treated queens (10\% FDR, Benjamini-Hochberg correction). Although imidacloprid has a unique mode of action relative to the compounds included in our pesticide treatment mixture, queens exposed to the 
Table 1 Concentrations and hazard quotients (HQ) for components of the pesticide cocktail

\begin{tabular}{|c|c|c|c|c|c|}
\hline Pesticide & Mode of action & Group & Median detection $(\mathrm{ppb})^{\mathrm{a}}$ & Target ppb & Contributing $\mathrm{HQ}$ \\
\hline Coumaphos & Acetylcholine esterase inhibitor & Acaricide & 943 & 314 & 53.00 \\
\hline tau-Fluvalinate & Sodium channel modulator & Acaricide & 4310 & 1437 & 332.56 \\
\hline 2,4-DMPF ${ }^{\mathrm{b}}$ & Octopamine receptor agonist & Acaricide (metabolite) & 304 & 101 & 1.35 \\
\hline Chlorothalonil & Multisite activity & Fungicide & 361 & 120 & 1.08 \\
\hline Chlorpyrifos & Acetylcholine esterase inhibitor & Insecticide & 2.7 & 1 & 11.81 \\
\hline Fenpropathrin & Sodium channel modulator & Insecticide & 16.8 & 6 & 112 \\
\hline Pendimethalin & Inhibition of microtubule assembly & Herbicide & 5.3 & 2 & 0.02 \\
\hline Atrazine & Inhibition of photosynthesis & Herbicide & 5.4 & 2 & .01 \\
\hline \multirow[t]{2}{*}{ Azoxystrobin } & Cytochrome bc1 inhibitor & Fungicide & 5.1 & 2 & .01 \\
\hline & & & Total HQ & & 511 \\
\hline
\end{tabular}

${ }^{a}$ Median wax concentrations and LD50s as determined by Traynor et al. (2016) [18]

${ }^{b}$ 2,4-Dimethylphenyl formamide (Amitraz degradate)

imidacloprid treatment yielded highly similar protein expression patterns when compared to cocktail-treated queens, and no proteins were differentially expressed between the two treatment groups. Despite showing the same trends as the cocktail treatments, only three proteins were differentially expressed between the imidacloprid and acetone groups, apparently owing to higher variability in the imidacloprid samples (Fig. $3 \mathrm{~b}$ ). A single protein, XP_625100.1 (a glyoxylase), was differentially expressed in both imidacloprid- and cocktail-treated queens relative to acetone queens. As expected, the GO term 'response to toxic substance,' driven by upregulated proteins, was significantly enriched among others (Fig. 3c). The top two most significantly upregulated proteins in the pesticide cocktail-treated queens were XP_026296889.1 (catalase, a well-known peroxidase) and XP_392368.1 (cytochrome c oxidase subunit 5a, the final complex in the electron transport chain).

\section{Expression patterns in failed and healthy queens}

To observe proteomic shifts in queens that failed in the field, we solicited samples of healthy $(n=45)$, imported

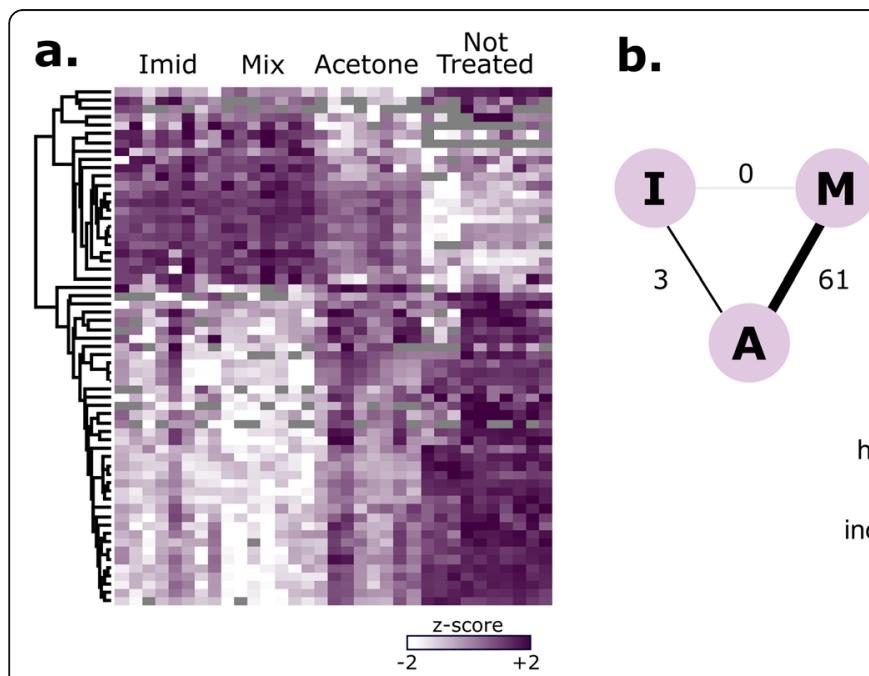

$$
\begin{array}{r}
\text { peroxiredoxin activity } \\
\text { C. } \\
\text { oxidoreductase activity, acting on peroxide- } \\
\text { structural constituent of cytoskeleton } \\
\text { GTPase activity } \\
\text { oxidoreductase activity } \\
\text { ribonucleoside binding } \\
\text { GTP binding } \\
\text { microtubule-based process } \\
\text { response to toxic substance } \\
\text { cellular response to toxic substance } \\
\text { ATP hydrolysis coupled proton transport } \\
\text { active transmembrane transporter activity } \\
\text { ATPase activity, coupled } \\
\text { nucleoside-triphosphatase activity } \\
\text { hydrolase activity, acting on acid anhydrides } \\
\text { pyrophosphatase activity } \\
\text { transmembrane transporter activity } \\
\text { inorganic molecule transmembrane transport } \\
\text { proton transmembrane transport } \\
\text { cation transport }
\end{array}
$$

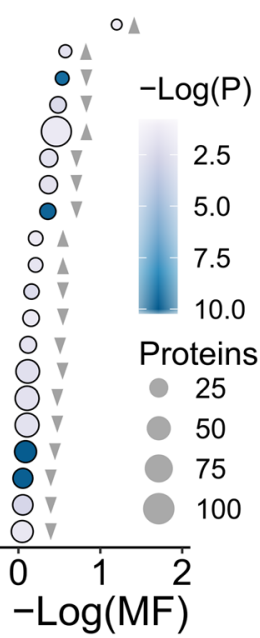

Fig. 3 Impact of direct contact pesticide exposure on queen spermathecal protein expression. a Protein expression changes induced by pesticide exposure and acetone (only proteins significantly different between the pesticide and acetone treatments, surviving 10\% FDR threshold by the Benjamini-Hochberg method, are shown). The pesticide treatment was a low-dose mixture of compounds (acaricides, fungicides, herbicides, and pesticides) found in the wax of honey bee colonies, blended in field-realistic proportions. See Table 1 for the relative components. The total dose each queen received was equivalent to a hazard quotient of 502 (imidacloprid) or 511 (mixture). Each column represents the spermatheca from one queen. $\mathbf{b}$ Schematic of differentially expressed proteins between groups ( $A=$ acetone, $I=$ imidacloprid, $M=$ mixture). $\mathbf{c} G O$ term enrichment analysis. Grey triangles indicate the direction of expression driving the enrichment (pesticide mixture relative to acetone). Only GO terms passing a 5\% false discovery cut-off are shown (Benjamini-Hochberg method). MF stands for multifunctionality score (higher values are less multifunctional). Circle size is proportional to the number of proteins belonging to the $\mathrm{GO}$ term 
( $n=18)$, and failed $(n=60)$ queens from beekeepers in British Columbia, Canada. Failed queens were defined as any queen whose reproductive capacity was not satisfactory to the beekeeper (e.g., symptoms include poor laying pattern, poor colony build-up, poor colony strength, and opportunistic diseases). We found a general upregulation of 139 proteins in failed queens (5\% FDR, Benjamini-Hochberg correction), and again the GO term 'response to toxic substance' was significantly enriched (Fig. 4a and b). Interestingly, the candidate markers, two for heat-shock and two for pesticide exposure, were significantly upregulated in failed queens relative to controls at a global scale, whereas the cold-shock markers were not (Fig. 4c; see Table 2 for summary statistics).

\section{Blind heat-shock trial}

To determine if we could use the heat-shock markers, XP_001120006.2 and XP_395659.1, to correctly identify heat-shocked queens despite no knowledge of their treatment group, we performed a blind heat-shock trial (Fig. 5). Setting the expression elevation threshold to 0.05 (only heat-shocked queens showed expression above this threshold in the previous unblind trial), eight of sixteen queens displayed elevated XP_001120006.2 (six of which were correctly assigned), whereas five queens displayed elevated XP_395659.1 (only two of which were correctly assigned). Therefore, $\mathrm{XP}_{-}$ 001120006.2 is a better biomarker, with $75 \%$ true positive rate, whereas XP_395659.1 performed poorly, with only $25 \%$ true positive rate.

\section{Discussion}

Here we describe progress made on identifying biomarkers for different forms of queen stress, which we ultimately aim to develop as a diagnostic test. We report new candidate markers proposed for cold-stress and

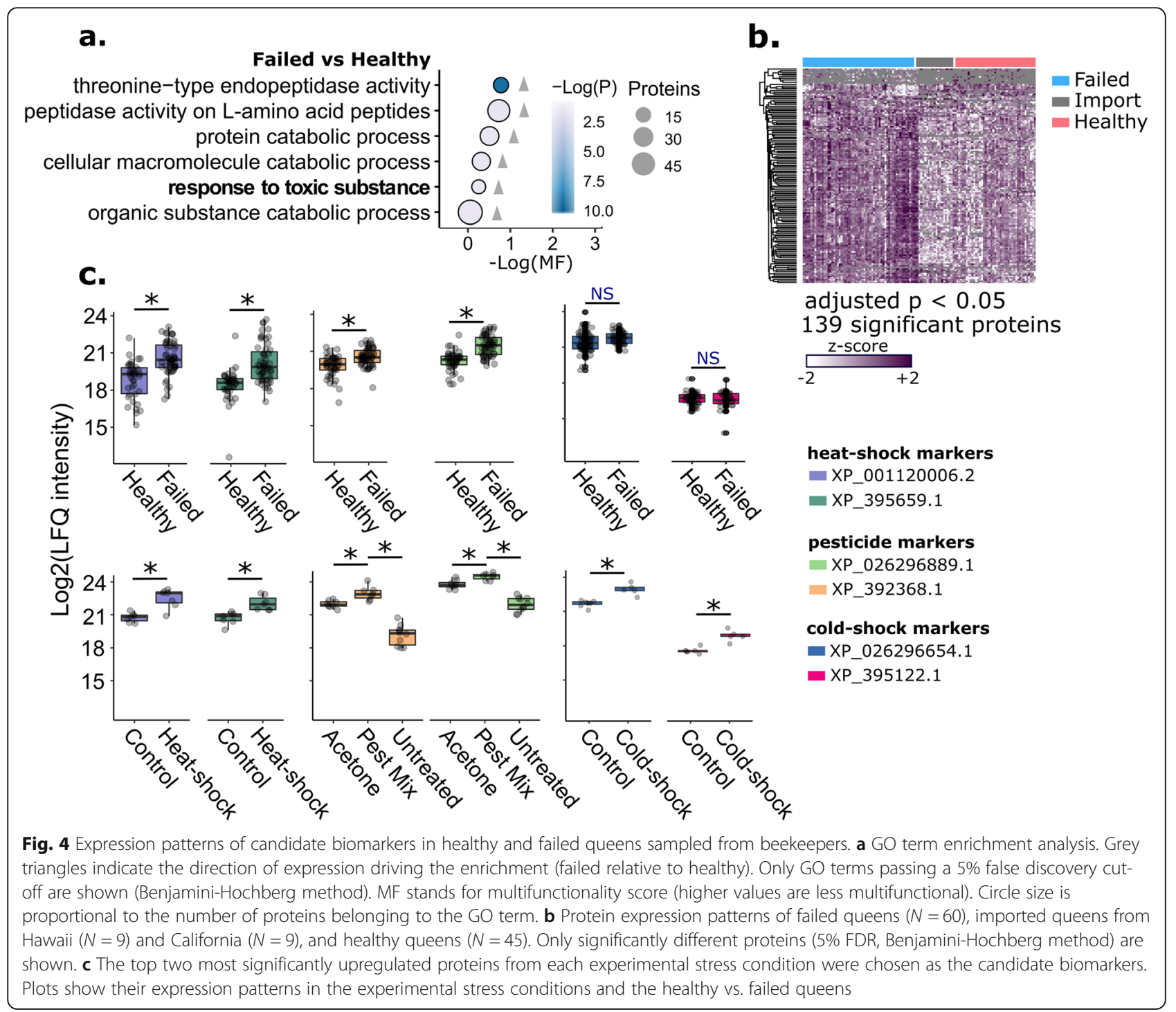


Table 2 Summary statistics for candidate stress biomarkers

\begin{tabular}{|c|c|c|c|c|c|}
\hline Accession & Stress & Comparison & Test statistic & $P$ & Adjusted P \\
\hline \multirow[t]{2}{*}{ XP_001120006.2 } & Heat & Heat-shock: Control & 2.89 & 0.00044 & 0.013 \\
\hline & & Failed:Healthy & 4.51 & $1.72 \times 10^{-5}$ & 0.0021 \\
\hline \multirow[t]{2}{*}{ XP_395659.1 } & Heat & Heat-shock: Control & 3.22 & 0.0015 & 0.022 \\
\hline & & Failed:Healthy & 5.26 & $8.50 \times 10^{-7}$ & 0.00024 \\
\hline \multirow[t]{2}{*}{ XP_026296889.1 } & Pesticide & Pest:Acetone & 3.9 & 0.00183 & 0.085 \\
\hline & & Failed:Healthy & 6.47 & $2.59 \times 10^{-9}$ & $3.27 \times 10^{-6}$ \\
\hline \multirow[t]{2}{*}{ XP_392368.1 } & Pesticide & Pest:Acetone & 4.54 & 0.000554 & 0.045 \\
\hline & & Failed:Healthy & 3.13 & 0.00234 & 0.045 \\
\hline \multirow[t]{2}{*}{ XP_026296654.1 } & Cold & Cold-shock: Control & 5.54 & 0.00025 & 0.042 \\
\hline & & Failed:Healthy & 2.29 & 0.024 & 0.17 \\
\hline \multirow[t]{2}{*}{ XP_395122.1 } & Cold & Cold-shock: Control & 5.63 & 0.00032 & 0.038 \\
\hline & & Failed:Healthy & 0.74 & 0.46 & 0.74 \\
\hline
\end{tabular}

pesticide-stress, and further develop those we have already proposed for heat-stress. Through this shot-gun discovery approach, we also gain some insight into how these stressors affect the biology of the spermatheca on a proteome-wide scale. Interestingly, some of these proposed markers are also upregulated in queens that failed passively in the field, indicating that they could have practical utility for the apiculture industry.

In our previous experiment, proposed candidate heatshock biomarkers by investigating queens which were at least third generation locals, all reared from a single mother colony. In the present study, we sourced queens from a commercial supplier in California. Since the same heat-shock proteins are consistently upregulated in queens of both origins, this suggests that these candidate biomarkers may be part of a broadly conserved heatshock response. However, several other geographically isolated queen sources contribute to North America's commercial supply, such as those from Chile, Australia, and Hawaii, which were not tested here. Furthermore, different stressors may or may not yield consistent biological responses among queens from different origins. Before these candidate biomarkers (for heat stress or otherwise) are widely used, they will need to be validated across a range of queen sources that are representative of the commercial industry.

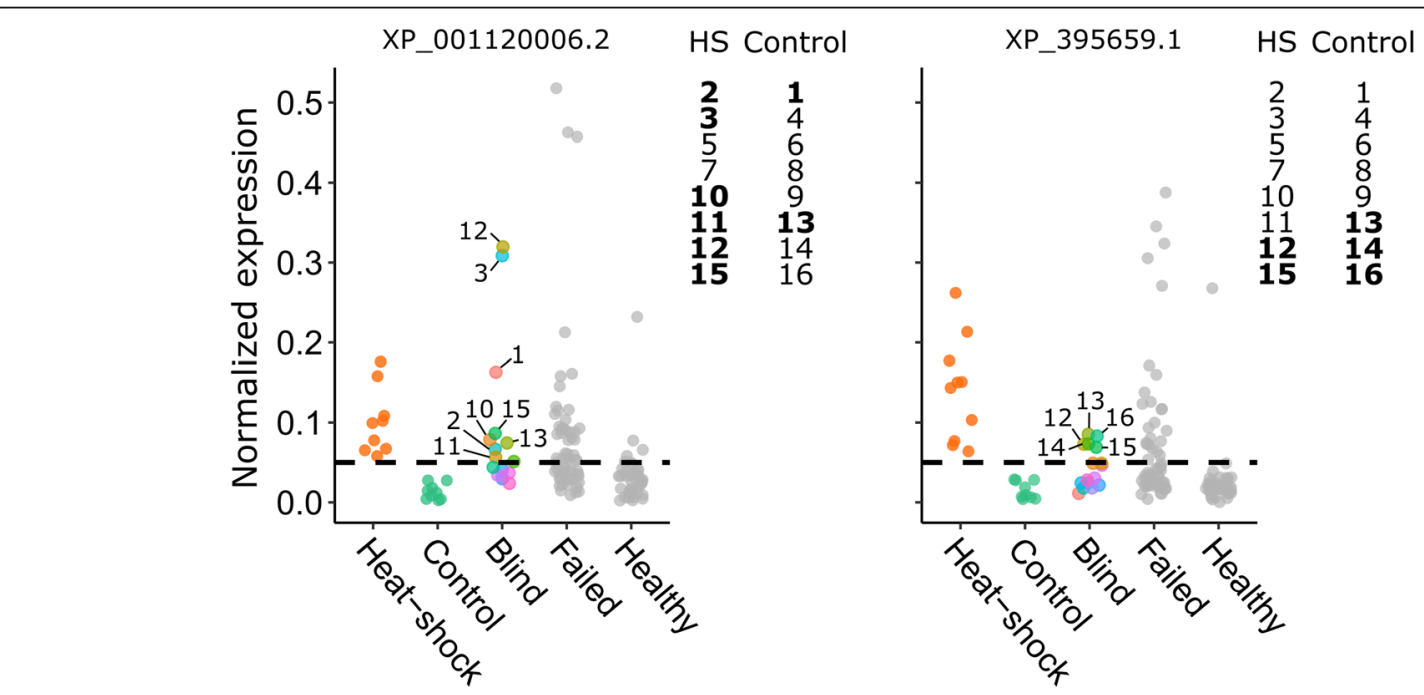

Fig. 5 Expression of candidate heat-shock biomarkers in a blind heat-shock trial. Protein expression (normalized LFQ intensity) was normalized to the protein with the lowest variance across all the spermatheca samples (XP_623495.1, V-type proton ATPase catalytic subunit). The heat-shock and control data were previously published. The blind queens were randomized into experimental groups (heat-shock (HS) and control) by a third party and groupings were only revealed after the data were plotted. Queens in the HS group were exposed to $40^{\circ} \mathrm{C}$ for $2 \mathrm{~h}$, whereas queens in the control group were held at $27^{\circ} \mathrm{C}$. The prediction accuracy of candidate marker XP_001120006.2 outperformed the candidate marker XP_395659.1 
Both cold-shock and heat-shock caused general downregulation of proteins involved in small molecule metabolism (e.g., carboxylic acids and amino acids) and oxidoreductase activity, whereas for cold-shock, proteins involved in lipid metabolic processes increased. It is unclear why fatty acid metabolism is favored by coldshock, as is the role of the two cold-shock biomarkers we proposed: leucine-rich repeat transmembrane neuronal protein 4-like (or LRRT-4, XP_026296654.1) and probable tRNA N6-adenosine threonylcarbamoyltransferase (XP_395122.1). LRRTs are best known for their roles in regulation and development of excitatory and inhibitory synapses [24]. However, our sample preparation method should not enable extraction of neuronal proteins nor transmembrane proteins (it is the soluble spermathecal fluid proteome), so this function is unlikely here. Indeed, inspecting the sequence using the Basic Local Alignment Search Tool (BLAST) shows that while the protein does have an LRR domain, most of the protein's sequence is devoted to an E3 ubiquitin-protein ligase domain (which tags proteins with ubiquitin, targeting them for degradation). Therefore, we think it is more likely that XP_026296654.1 is involved in the downregulation of oxidoreductases and small molecule catabolic enzymes that we observe with cold-shock. It is not clear what the relationship is between the threonylcarbamoyltransferase enzyme (which, as the name suggests, adds a threonylcarbamoyl group to specific tRNAs) and cold-shock. But, overall, these results are consistent with temperature stress generally causing metabolic dysregulation in the spermatheca, which may contribute to how heat-and cold-shock leads to sperm death.

The imidacloprid pesticide treatment was originally included only as a positive control, since contact with such a large dose inside a colony is highly unlikely (and therefore, we did not aim to find queen biomarkers for exposure to this pesticide). We expected the imidacloprid treatment to have the greatest effect on protein expression; however, the expression patterns of the imidacloprid-treated queens were remarkably similar to the cocktail-treated queens. This is despite the cocktail not containing any imidacloprid (indeed, it does not contain any neonicotinoid insecticides), and accounting for the effect of the carrier (acetone) by making statistical comparisons only to acetone-only controls (and not untreated queens). Our data, therefore, show a generalized detoxification response that was unselective when compared between a compound with a distinctive mode of action (imidacloprid) and a nine-component pesticide mixture. It could be that other tissues (e.g., the fat body and hemolymph) are the major sites of detoxification from contact exposures, and the changes observed in the spermathecal fluid proteome are mainly secondary effects. Indeed, while 'response to toxic substance' was a significantly enriched GO term, other GO terms were far more significant ('structural constituent of cytoskeleton,' 'microtubule-based process,' and 'transmembrane transporter activity') were highly significantly enriched via protein downregulation.

Catalase, which was upregulated in pesticide-treated queens, is well known for its role in mitigating peroxideinduced oxidative stress, and its upregulation has been previously linked to pesticide exposure in other insects (e.g. Harmonia axyridis, Sogatella furcifera, Diadegma semiclausum) [25-27]. This is likely because pesticide detoxification often leads to oxidative stress, which must be counteracted to avoid DNA damage and cell death [28-30]. The observation that not only catalase, but also other enzymes contributing to the 'response to toxic substance' GO term were upregulated in both the pesticide-treated and the failed queens suggests that failed queens may suffer from increased exposure to environmental toxins, possibly through chronic lifetime contact exposure to pesticides in wax. Although the exact causes of failure for these queens are ultimately unknown, these observations are consistent with previous work linking queen failure, reduced brood production, and loss of sperm viability to pesticide exposure (indeed, these failed queens also had significantly lower sperm viability compared to healthy queens, as we previously reported) $[5,7,21,31]$.

Although catalase was also upregulated in failed queens as well as experimentally pesticide-stressed queens, this does not necessarily mean that the failed queens were pesticide stressed. Similarly, although the candidate heat-shock markers were upregulated in failed queens, at this stage we cannot say definitively if this was related to heat. This is because the failed queens also tended to be older than the healthy queens, and because of this sampling bias, we cannot confidently distinguish between proteins changing as a result of age, and those changing with health status. However, even if catalase expression is mainly influenced by age, that would also be an interesting result. In other species (including other insects), catalase expression is linked to fecundity and is thought to help protect the developing oocyte from oxidative damage [32]. Stored sperm require similar protection from oxidative stress (indeed, honey bees have evolved other strategies, such as a highly anaerobic spermathecal environment, to help achieve this) [33-35]. But catalase expression typically declines with age, along with fecundity [32], which is not the trend we observed here. If honey bee queens have instead evolved to upregulate catalase with age (with or without added pesticide stress), this could help explain how they maintain high fecundity throughout their long lives, through improved protection of oocytes and sperm cells from oxidative 
damage. Indeed, catalase expression appears to be tightly linked to sperm maintenance, as the enzyme becomes significantly upregulated in the spermatheca during the transition from virgin to mated queen [7, 34, 35]. More experiments will be necessary to determine whether catalase expression correlates with age independently of queen health status.

In the blind heat-shock trial, we were surprised that the candidate biomarkers (the small heat-shock proteins (HSPs) XP_001120006.2 and XP_39659.1) were not more useful for classifying queens into their respective treatment groups. We expected that queens with both proteins significantly upregulated would be, most confidently, the ones that were heat-shocked; however, of the three queens with both proteins upregulated, only two were actually heat-shocked. Therefore, this method has lower true positive rate (25\% compared to $75 \%$ ) than using XP_001120006.2 alone. Although a 75\% true positive rate is better than chance, it is still not satisfactory for a diagnostic test. For the test to be practically useful, we aim to explore different methods of data acquisition and normalization to minimize noise, as well as determine the biological limits of marker utility (e.g., how long after heat-shock the marker remains upregulated).

Important biomarker attributes remain to be determined, especially for the cold-stress and pesticide-stress candidates, which are yet lacking dose-response tests. For the markers to be practically useful, the minimum stress threshold leading to marker activation needs to be determined (similar to how we determined that heat-stress for $>1 \mathrm{~h}$ is necessary to activate the sHSP markers, here) through dose-response experiments. Such experiments would also help identify if, for example, in extreme cases, new, more determinant biomarkers are activated. Likewise, their practical utility will also be influenced by the time window between which the markers are activated and when they decline back to basal levels (if ever), especially considering that the main test subjects will be failed queens - a phenotype which can take months post-stress to observe. For factors such as pesticide stress, where queens are likely chronically exposed, such a decline in marker expression may not even occur. However, it is not known exactly how much exposure queens experience from chronic contact with contaminated wax. For heat stress, we anticipate that candidate biomarkers would be upregulated initially and then decrease over time, but the general upregulation of candidate heat stress markers in failed queen suggests that either these markers are longerlasting than expected, or another, yet unidentified stress also causes upregulation of these specific proteins.

\section{Conclusion}

Heat-shock, cold-shock, and pesticide exposure induce unique proteomic stress signatures in honey bee queen spermathecae. In addition to offering biological insights into how these stressors alter the spermathecal environment, and providing candidate proteins to investigate as potential causal factors for stress-associated reductions in sperm viability, these signatures may also be useful as biomarkers for specific causes of queen failure. Some of these protein expression patterns are also apparent in queens that failed in the field, but due to sampling bias, we cannot determine the extent to which these expression patterns are linked to failure, and the extent to which they are linked to age. Our blind heat-shock trial shows that markers with similar stress-associated expression patterns can have markedly different predictive power, demonstrating that each marker will need to be individually optimized and validated before they can be used as a reliable diagnostic test.

\section{Methods}

\section{Queen sources}

Queens for temperature-stress tests were shipped to Vancouver, Canada, on July 3rd, 2019, from a commercial supplier in California. Queens for the pesticidestress experiments were shipped on April 24th, 2019, from a commercial supplier in Chile. Immediately upon arrival, queens and attendants were given a few drops of water and held overnight at $30^{\circ} \mathrm{C}$ before exposure to their respective stressors.

Queens for the survey of failed and healthy queens were obtained from local beekeepers and queen breeders in British Columbia, Canada (Kettle Valley Queens, Nicola Valley Honey, Wild Antho, Campbells Gold Honey, Heather Meadows Honey Farm, Six Legs Good Apiaries, Wildwood Queens, Cariboo Honey, and Worker Bee Honey Company) between the first week of June 5th and July 10th, 2019. See Table S1 for supplementary queen information and descriptions (such as shipment method, mating day, lineage, etc.). Queens were shipped with temperature loggers (WatchDog B1000 series) to verify that there was not adverse exposure during transport. 'Healthy' queens were all evaluated to have a good laying pattern by the beekeepers (consistent egg distribution, few missed cells, one egg per cell) and were 2 months old. 'Failed' queens were rated as inferior by beekeepers on the basis of symptoms such as poor colony population build-up, drone-laying, and sparse brood patterns [36]. Some were failing due to diseases such as chalkbrood or had EFB-like symptoms, and it was not clear if this was an effect of queen quality or some other factor. We previously verified that failed queens had significantly lower sperm viability than healthy queens [6]. Failed queens tended to be older than healthy queens, and ages of some were unknown. Imported queens were obtained from Hawaii $(n=9)$ and California $(n=9)$, shipped by international air, arriving on June 14th, 2019. 


\section{Stressing queens}

For the time-course heat-shock experiment, queens were held in an incubator set to $40{ }^{\circ} \mathrm{C}$ and $40 \% \mathrm{RH}$ for 0.25 , $0.5,1$, or $2 \mathrm{~h}$ ( $n=7$ each). Another group of queens $(n=$ 6) were held at $40{ }^{\circ} \mathrm{C}$ for $2 \mathrm{~h}$ at $80 \% \mathrm{RH}$ to test for a humidity effect. For the cold-shock experiment, queens $(n=5)$ were held at $4{ }^{\circ} \mathrm{C}$ for $2 \mathrm{~h}$, then $30^{\circ} \mathrm{C}$ for $2 \mathrm{~d}$. Control queens $(n=7)$ were not exposed to heat, but were held in the same $30^{\circ} \mathrm{C}$ incubator for 2 days with the other queens.

Queens for the pesticide-stress experiments were first anesthetized with carbon dioxide, then $2 \mu \mathrm{l}$ of acetone containing either nothing, $20 \mathrm{ppb}$ imidacloprid (Chem Service Inc., West Chester, PA), 97.9\% purity, 20 ppb produced via serial dilution in acetone), or a mix of nine different pesticides listed in Table 1 , yielding a cumulative HQ of 502, was dispensed onto the thorax of each queen ( $n=8,8$, and 7 , respectively). All compounds in the pesticide mixture were purchased as pure technical material ( $\geq 95 \%$ purity) from Sigma Aldrich (St. Louis, $\mathrm{MO})$ or Chem Service Inc. and were serially diluted in acetone in order to achieve the respective concentrations listed in Table 1. An additional group of $n=10$ queens were untreated. Queens were then held at $30^{\circ} \mathrm{C}$ for $2 \mathrm{~d}$ prior to dissecting and freezing their spermathecae.

\section{Blind heat-shock trial}

Queens for the blind heat-shock experiment were removed from active hives near Kutztown, PA and were 1-6 months of age and actively laying. The colonies were rated as being in good health with no visual signs of disease and good brood pattern. Queens were placed in queen cages with attendants and food and transported via automobile to NC State University within 2 days of being removed from colonies. Queens were placed inside the aluminum block well of a dry heat bath with cover and held for $2 \mathrm{~h}$ at $40^{\circ} \mathrm{C}$. Queens were then held in an incubator for an additional $48 \mathrm{~h}$ at $30{ }^{\circ} \mathrm{C}$ and then frozen at $-80^{\circ} \mathrm{C}$ and shipped on dry ice to British Columbia, Canada.

\section{Proteomics sample preparation}

Protein was extracted from spermathecae by lysing the organ in $100 \mu \mathrm{l}$ Buffer D (17 mM D-glucose, $54 \mathrm{mM} \mathrm{KCl}$, $25 \mathrm{mM} \mathrm{NaHCO} 3,83 \mathrm{mM} \mathrm{Na}_{3} \mathrm{C}_{6} \mathrm{H}_{5} \mathrm{O}_{7}$ ). Sperm cells and tissue debris were spun down by centrifuging tubes at $2000 \mathrm{~g}$ for $5 \mathrm{~min}$, then pipetting off the top $80 \mu \mathrm{l}$ of supernatant. The supernatant was diluted 1:1 with distilled water, then proteins were precipitated by adding four volumes of ice-cold $100 \%$ acetone and incubating at $-20{ }^{\circ} \mathrm{C}$ overnight. Precipitated proteins were spun down $\left(10,000 \mathrm{~g}\right.$ for $\left.10 \mathrm{~min}, 4^{\circ} \mathrm{C}\right)$ and the supernatant was removed. The protein pellets were washed twice with $500 \mu \mathrm{l}$ ice-cold $80 \%$ acetone, dried at room temperature for $15 \mathrm{~min}$, then solubilized in urea digestion buffer $(6 \mathrm{M}$ urea, $2 \mathrm{M}$ thiourea, $100 \mathrm{mM}$ Tris, $\mathrm{pH}$ 8.0) and processed for proteomics analysis as previously described [7]. Briefly, protein samples were reduced $(1 \mu \mathrm{g}$ dithiothreitol), alkylated (5 $\mu \mathrm{g}$ iodoacetamide, dark), and digested (1 $\mu \mathrm{g}$ Lys-C, $4 \mathrm{~h}$, then $1 \mu \mathrm{g}$ Trypsin, overnight) at room temperature. Peptides were acidified, then desalted using in-house packed C18 STAGE tips, quantified using a nanodrop (A280 nm), and $1 \mu \mathrm{g}$ of peptides were analyzed in random order by liquid chromatographytandem mass spectrometry (LC-MSMS; Thermo EasynLC 1000 coupled to a Bruker Impact II quadrupole time-of-flight mass spectrometer) [37], using parameters exactly as previously described [7].

As stated in McAfee et al. [7], the LC system included a fused-silica (5- $\mu \mathrm{m}$ Aqua C18 particles (Phenomenex)) fritted $2-\mathrm{cm}$ trap column connected to a $50-\mathrm{cm}$ analytical column packed with ReproSil C18 (3- $\mu$ m C18 particles (Dr. Maisch)). The separation gradient ran from 5 to $35 \%$ Buffer B (80\% acetonitrile, $0.1 \%$ formic acid) over $90 \mathrm{~min}$, followed by a $15 \mathrm{~min}$ wash at $95 \%$ Buffer B (flow rate: $250 \mu \mathrm{l} / \mathrm{min}$ ). The instrument parameters were: scan from 150 to $2200 \mathrm{~m} / \mathrm{z}, 100 \mu \mathrm{s}$ transient time, $10 \mu \mathrm{s}$ prepulse storage, $7 \mathrm{eV}$ collision energy, $1500 \mathrm{Vpp}$ Collision $\mathrm{RF}, \mathrm{a}+2$ default charge state, $18 \mathrm{~Hz}$ spectral acquisition rate, 3.0 s cycle time and the intensity threshold was 250 counts.

Instrument performance tests were performed weekly after routine maintenance by infusing a low concentration tuning mixture (Agilent), diluted 1:5 in Buffer A, plus glu-1-fibrinopeptide (200 pmol/ml), and assessing ion funnel transmission, quadrupole transmission, collision cell dynamic performance and collision cell transmission according to the manufacturer's protocol (Bruker HDX Solution). If performance fell below the manufacturer's limits, the instrument was cleaned and the test was repeated. An in-house prepared HeLa cell digest is also injected after weekly maintenance and inspected for richness and number of protein identifications (typically 2000 protein groups from a 45 min LC gradient). Bovine serum albumin (BSA) peptides were injected and analyzed between approximately every 10 samples to assess the peak width and mass accuracy of the instrument. If either HeLa or BSA results were not satisfactory, LC maintenance was performed.

\section{Mass spectrometry data processing}

After brief inspection for chromatogram richness and peak shape, raw mass spectrometry data files were searched with MaxQuant ( $v$ 1.6.1.0) [38] using default parameters except label-free quantification was enabled, match between runs was enabled, and the LFQ min ratio count was set to 1 . Complete search parameters can be found within the mqpar.xml file within the Proteome 
Exchange repository. The Apis mellifera reference proteome database was downloaded from NCBI on November 18th, 2019 (HAv3.1) and all honey bee virus sequences were added to the fasta file. The final search database is also available in the data repository. All proteomics raw data, search results, and search parameters for the failed, healthy, and imported queens are hosted by MassIVE (www.massive.ucsd.edu; accession: MSV000085056). Data for temperature exposure duration, the blind heatshock trial, pesticide, and cold-shock data has the accession MSV000085041. Within experiments, average mass errors were $0.92 \mathrm{ppm}$ (multiple stressor experiment), $0.91 \mathrm{ppm}$ (temperature time course experiment), 1.24 ppm (blind heat-shock trial), and $0.90 \mathrm{ppm}$ (failed and healthy queens). Detailed mass spectrometry data quality metrics are available in the summary files within the MaxQuant output folders, which are included in the MassIVE repositories.

\section{Differential expression analysis}

Differential expression analysis for the experimentally stressed queens was performed using Perseus (v 1.5.6.0) as previously described [7]. Briefly, data were $\log 2$ transformed, then reverse hits, proteins only identified by site, and contaminants were removed, along with any protein identified in fewer than six samples. Samples were compared in pairwise combinations (heat-shock to control, cold-shock to control, and pesticide-stressed to acetone) using t-tests with a Benjamini-Hochberg multiple hypothesis testing correction to 5\% FDR (for heat-shock and cold-shock experiments) or 10\% FDR (for the pesticide-stress experiment). For the pesticide experiments, we used acetone-treated queens as the negative control because we were interested in genes that were influenced by the pesticides specifically, over and above the effect of the solvent. However, we still evaluated expression in untreated queens in order to confirm that proteins differentially expressed between acetone and pesticide queens were also differentially expressed between pesticide queens and untreated queens, and in the same direction (representing the more natural condition). For this experiment, we relaxed the FDR because at 5\% FDR there were not two proteins significantly upregulated compared to acetone samples which were also identified in the healthy vs. failed queen survey.

The queen survey data is part of a larger project (yet unpublished) evaluating relationships between spermathecal protein expression and other queen phenotypes; therefore, these data were analyzed with a more sophisticated linear model with multiple fixed continuous and discrete covariates. To do this, we used the limma package in $\mathrm{R}$ [39]. The results of this analysis were also corrected to 5\% FDR by the BenjaminiHochberg method.

\section{GO term enrichment analysis}

Gene ontology (GO) terms were retrieved for protein groups using BLAST2GO ( $\mathrm{v} 4.0$ ) and enrichment tests were conducted using the gene score resampling method within ErmineJ [23]. This method operates independently of a 'background' and 'hit list' of significantly differentially expressed proteins, and is therefore not sensitive to sliding FDR thresholds of up-stream differential expression analyses. Rather, it reads in raw $p$ values and calculates if proteins with low $\mathrm{p}$ values are more likely to share GO terms than expected by chance, along a continuous scale. More documentation about the gene score resampling method can be found on the software developer's website (https:// erminej.msl.ubc.ca/) [23]. For each experimental condition, we performed enrichment analyses on upregulated and downregulated proteins separately. The enrichment FDR was also adjusted to $5 \%$ using the BenjaminiHochberg method.

\section{Supplementary information}

Supplementary information accompanies this paper at https://doi.org/10. 1186/s12864-020-06992-2.

\section{Additional file 1}

\section{Abbreviations}

BLAST: Basic local alignment search tool; DMPF: 2,4-Dimethylphenyl-N '-methyl-formamidine (degradate of Amitraz); FDR: False discovery rate; GO: Gene ontology; HQ: Hazard quotient; HSP: Heat-shock protein; LCMSMS: Liquid chromatography tandem mass spectrometry; LFQ: Label-free quantification; LRRT protein: Leucine-rich repeat transmembrane protein; MF: Multifunctionality; RH: Relative humidity

\section{Acknowledgements}

We thank Kettle Valley Queens, Nicola Valley Honey, Wild Antho, Campbells Gold Honey, Heather Meadows Honey Farm, Six Legs Good Apiaries, Wildwood Queens, Cariboo Honey, and Worker Bee Honey Company for donating failed and healthy queens for this research. We also thank Robyn Underwood for supplying the queens used for the blind heat-shock test, and Heather Higo, Marta Guarna, and Liz Huxter for organizing the BC queen survey.

\section{Authors' contributions}

AM wrote the manuscript, designed the experiments, prepared proteomics samples, and analyzed the proteomics data. AC coordinated and received queens for the queen survey and dissected queens for the stress tests. JM produced the pesticide cocktail, helped design the pesticide stress experiment, and assisted with data analysis and interpretation. JSP conducted the blind heat-shock trial. Grants to AM, LJF, JSP, and DRT funded the research. The author(s) read and approved the final manuscript.

\section{Funding}

This work was funded from a Project Apis $m$ grant awarded to AM and LJF an NSERC Discovery grant (311654-11), and funding from Genome Canada and Genome British Columbia (project 214PRO) awarded to LJF, and a USDA-NIFA grant (2016-07962) awarded to JSP and DRT.

\section{Availability of data and materials}

The datasets and databases generated and/or analysed during the current study are hosted by MassIVE (www.massive.ucsd.edu) and are available at ftp://MSV000085056@massive.ucsd.edu. Data for temperature exposure duration, the blind heat-shock trial, pesticide, and cold-shock data is available at ftp://MSV000085041@massive.ucsd.edu. 


\section{Ethics approval and consent to participate}

As a non-cephalopod invertebrate species, honey bees are not subject to animal ethics committee approval at the University of British Columbia.

\section{Consent for publication}

Not applicable.

\section{Competing interests}

JSP owns a honey bee consulting business. All other authors declare no competing financial interests.

\section{Author details}

'Department of Entomology \& Plant Pathology, North Carolina State University, Raleigh, North Carolina, USA. ${ }^{2}$ Department of Biochemistry and Molecular Biology, Michael Smith Laboratories, University of British Columbia, Vancouver, British Columbia, Canada. ${ }^{3}$ Pettis and Associates LLC, Salisbury, MD, USA.

Received: 28 February 2020 Accepted: 13 August 2020

Published online: 20 August 2020

\section{References}

1. CAPA statement on honey bee wintering colony losses in Canada [http://www.capabees.com/shared/2018-2019-CAPA-Statement-onColony-Losses.pdf].

2. VanEngelsdorp D, Hayes J, Underwood RM, Pettis J, Gay N. A survey of honey bee colony losses in the US, fall 2007 to spring 2008; 2008.

3. Kulhanek K, Steinhauer N, Rennich K, Caron DM, Sagili RR, Pettis JS, Ellis JD, Wilson ME, Wilkes JT, Tarpy DR. A national survey of managed honey bee 2015-2016 annual colony losses in the USA. J Apic Res. 2017;56(4):328-40.

4. Pettis JS, Rice N. Joselow K, vanEngelsdorp D, Chaimanee V: Colony failure linked to low sperm viability in honey bee (Apis mellifera) Queens and an exploration of potential causative factors. PLoS One. 2016;11(2):e0147220.

5. Chaimanee V, Evans JD, Chen Y, Jackson C, Pettis JS. Sperm viability and gene expression in honey bee queens (Apis mellifera) following exposure to the neonicotinoid insecticide imidacloprid and the organophosphate acaricide coumaphos. J Insect Physiol. 2016:89:1-8.

6. Stabentheiner A, Kovac H, Brodschneider R. Honeybee colony thermoregulation-regulatory mechanisms and contribution of individuals in dependence on age, location and thermal stress. PLoS One. 2010;5(1):e8967.

7. McAfee A, Chapman A, Higo H, Underwood R, Milone J, Foster LJ, Guarna MM, Tarpy DR, Pettis JS. Vulnerability of honey bee queens to heat-induced loss of fertility. Nature Sustainability. 2020;3:367-76.

8. Withrow JM, Pettis JS, Tarpy DR. Effects of temperature during package transportation on queen establishment and survival in honey bees (Hymenoptera: Apidae). J Econ Entomol. 2019;112(3):1043-9.

9. Starks PT, Gilley DC. Heat shielding: a novel method of colonial thermoregulation in honey bees. Naturwissenschaften. 1999:86(9):438-40.

10. Villa JD, Gentry C, Taylor OR. Preliminary observations on thermoregulation, clustering, and energy utilization in African and European honey bees. J Kansas Entomol Soc. 1987;60(1):4-14.

11. Luber G, McGeehin M. Climate change and extreme heat events. Am J Prev Med. 2008;35(5):429-35.

12. Meehl GA, Tebaldi $C$. More intense, more frequent, and longer lasting heat waves in the 21st century. Science. 2004;305(5686):994-7.

13. Mao W, Schuler MA, Berenbaum MR. A dietary phytochemical alters casteassociated gene expression in honey bees. Sci Adv. 2015;1(7):e1500795.

14. Winston ML. The biology of the honey bee: Harvard university press; 1991.

15. Haydak MH. Honey bee nutrition. Annu Rev Entomol. 1970;15(1):143-56.

16. Lucchetti MA, Kilchenmann V, Glauser G, Praz C, Kast C. Nursing protects honeybee larvae from secondary metabolites of pollen. Proc R Soc B Biol Sci. 2018;285(1875):20172849

17. Böhme F, Bischoff $G$, Zebitz CP, Rosenkranz P, Wallner K. From field to food-will pesticide-contaminated pollen diet lead to a contamination of royal jelly? Apidologie. 2018;49(1):112-9.

18. Traynor KS, Pettis JS, Tarpy DR, Mullin CA, Frazier JL, Frazier M. In-hive pesticide Exposome: assessing risks to migratory honey bees from in-hive pesticide contamination in the eastern United States. Sci Rep. 2016;6:33207.

19. Calatayud-Vernich P, Calatayud F, Simó E, Picó Y. Pesticide residues in honey bees, pollen and beeswax: assessing beehive exposure. Environ Pollut. 2018; 241:106-14.
20. Stoner K, Eitzer B. Using a Hazard quotient to evaluate pesticide residues detected in pollen trapped from honey bees (Apis mellifera) in Connecticut. PLOS One. 2013;8(10):e77550.

21. Tsvetkov N, Samson-Robert O, Sood K, Patel HS, Malena DA, Gajiwala PH, Maciukiewicz P, Fournier V, Zayed A. Chronic exposure to neonicotinoids reduces honey bee health near corn crops. Science. 2017;356(6345):1395-7.

22. Sandrock C, Tanadini M, Tanadini LG, Fauser-Misslin A, Potts SG, Neumann P. Impact of chronic neonicotinoid exposure on honeybee colony performance and queen supersedure. PLoS One. 2014;9(8):e103592.

23. Lee HK, Braynen W, Keshav K, Pavlidis P. ErmineJ: tool for functional analysis of gene expression data sets. BMC Bioinformatics. 2005;6:269.

24. de Wit J, Ghosh A. Control of neural circuit formation by leucine-rich repeat proteins. Trends Neurosci. 2014:37(10):539-50.

25. Zhou C, Yang H, Wang Z, Long G-Y, Jin D-C. Protective and detoxifying enzyme activity and ABCG subfamily gene expression in Sogatella furcifera under insecticide stress. Front Physiol. 2019;9:1890.

26. Yang Q, Wang S, Zhang W, Yang T, Liu Y. Toxicity of commonly used insecticides and their influences on protective enzyme activity of multicolored Asian lady beetle Harmonia axyridis (Pallas). J Plant Prot. 2015; 42(2):258-63.

27. Jia B, Hong S, Zhang Y, Cao Y. Effect of sublethal concentrations of abamectin on protective and detoxifying enzymes in Diagegma semclausum. J Environ Entomol. 2016;38(5):990-5.

28. Chakrabarti P, Rana S, Sarkar S, Smith B, Basu P. Pesticide-induced oxidative stress in laboratory and field populations of native honey bees along intensive agricultural landscapes in two eastern Indian states. Apidologie. 2015;46(1):107-29

29. Xu W, Yang M, Gao J, Zhang Y, Tao L. Oxidative stress and DNA damage induced by spinosad exposure in Spodoptera frugiperda Sf9 cells. Food Agric Immunol. 2018;29(1):171-81.

30. James $\mathrm{R}, \mathrm{Xu}$ J. Mechanisms by which pesticides affect insect immunity. J Invertebr Pathol. 2012;109(2):175-82.

31. Wu-Smart J, Spivak M. Sub-lethal effects of dietary neonicotinoid insecticide exposure on honey bee queen fecundity and colony development. Sci Rep. 2016:6:32108.

32. DeJong RJ, Miller LM, Molina-Cruz A, Gupta L, Kumar S, Barillas-Mury C. Reactive oxygen species detoxification by catalase is a major determinant of fecundity in the mosquito Anopheles gambiae. Proc Natl Acad Sci U S A. 2007;104(7):2121-6.

33. Paynter E, Millar AH, Welch M, Baer-Imhoof B, Cao D, Baer B. Insights into the molecular basis of long-term storage and survival of sperm in the honeybee (Apis mellifera). Sci Rep. 2017;7:40236.

34. Collins A, Williams V, Evans J. Sperm storage and antioxidative enzyme expression in the honey bee, Apis mellifera. Insect Mol Biol. 2004;13(2):141-6.

35. Baer B, Eubel H, Taylor NL, O'Toole N, Millar AH. Insights into female sperm storage from the spermathecal fluid proteome of the honeybee Apis mellifera. Genome Biol. 2009;10(6):R67.

36. Lee KV, Goblirsch M, McDermott E, Tarpy DR, Spivak M. Is the brood pattern within a honey bee colony a reliable indicator of queen quality? Insects. 2019;10(1):12.

37. Beck S, Michalski A, Raether O, Lubeck M, Kaspar S, Goedecke N, Baessmann C, Hornburg D, Meier F, Paron I, et al. The impact II, a very high-resolution Quadrupole time-of-flight instrument (QTOF) for deep shotgun proteomics. Mol Cell Proteomics. 2015;14(7):2014-29.

38. Cox J, Hein MY, Luber CA, Paron I, Nagaraj N, Mann M. Accurate proteomewide label-free quantification by delayed normalization and maximal peptide ratio extraction, termed MaxLFQ. Mol Cell Proteomics. 2014;13(9): 2513-26.

39. Ritchie ME, Phipson B, Wu D, Hu Y, Law CW, Shi W. Smyth GK: limma powers differential expression analyses for RNA-sequencing and microarray studies. Nucleic Acids Res. 2015:43(7):e47.

\section{Publisher's Note}

Springer Nature remains neutral with regard to jurisdictional claims in published maps and institutional affiliations. 